\title{
SOME INFINITE SERIES
}

\section{H. F. SANDHAM}

1. We sum eight general infinite series [1], particular cases of which are

1.11

$$
\frac{1^{3}}{\cosh (\pi / 2)}-\frac{3^{3}}{\cosh (3 \pi / 2)}+\frac{5^{3}}{\cosh (5 \pi / 2)}
$$

1.12

$$
-\frac{7^{3}}{\cosh (7 \pi / 2)}+\cdots=0 \text {, }
$$

1.22

$$
\frac{1}{1^{5} \cosh (\pi / 2)}-\frac{1}{3^{5} \cosh (3 \pi / 2)}+\frac{1}{5^{5} \cosh (5 \pi / 2)}
$$

$$
-\frac{1}{7^{5} \cosh (7 \pi / 2)}+\cdots=\frac{\pi^{5}}{768}
$$$$
\frac{1^{5}}{e^{\pi}+1}+\frac{3^{5}}{e^{3 \pi}+1}+\frac{5^{5}}{e^{5 \pi}+1}
$$

$$
+\frac{7^{5}}{e^{7 \pi}+1}+\cdots=\frac{31}{504}
$$

$$
\frac{\tanh (\pi / 2)}{1^{3}}+\frac{\tanh (3 \pi / 2)}{3^{3}}+\frac{\tanh (5 \pi / 2)}{5^{3}}
$$

1.31

1.32

$$
+\frac{\tanh (7 \pi / 2)}{7^{3}}+\cdots=\frac{\pi^{3}}{32}
$$

$$
\frac{1}{\sinh \pi}-\frac{2}{\sinh 2 \pi}+\frac{3}{\sinh 3 \pi}
$$

$$
-\frac{4}{\sinh 4 \pi}+\cdots=\frac{1}{4 \pi}
$$

$$
\frac{1}{1^{5} \sinh \pi}-\frac{1}{2^{5} \sinh 2 \pi}+\frac{1}{3^{5} \sinh 3 \pi}
$$

1.41

$$
\begin{aligned}
& -\frac{1}{4^{5} \sinh 4 \pi}+\cdots=\frac{\pi^{3}}{360}, \\
& \sum_{1}^{\infty} \frac{n^{13}}{e^{2 \pi n}-1}=\frac{1}{24}
\end{aligned}
$$

Received by the editors June 16, 1953. 


$$
\sum_{1}^{\infty} \frac{\operatorname{coth} \pi n}{n^{7}}=\frac{19 \pi^{7}}{56700}
$$

Noting that

$$
\frac{1}{e^{x}+1}=\frac{1}{2}-\frac{1}{2} \tanh \frac{x}{2}, \quad \frac{1}{e^{x}-1}=\frac{1}{2} \operatorname{coth} \frac{x}{2}-\frac{1}{2},
$$

it will readily be seen that each of the above four pairs of series contains one of the hyperbolic functions: sech, tanh, cosech, coth, multiplied by a positive and by a negative power. If we include the remaining two functions: $\sinh$ and cosh, we have of course two pairs of series diverging to infinity.

The general series are (3.22), (3.32); (4.22), (4.32); (5.22), (5.32); (6.222), (6.32).

2. The bases of the proofs are the partial fraction expansions

$$
\frac{1}{e^{2 \pi x}-1}=\frac{1}{2 \pi x}-\frac{1}{2}+\frac{1}{\pi} \sum_{1}^{\infty} \frac{x}{x^{2}+n^{2}}
$$

$$
\frac{e^{2 \pi a x}}{e^{2 \pi x}-1}=\frac{1}{2 \pi x}+\frac{1}{\pi} \sum_{1}^{\infty}\left\{\frac{x \cos 2 n \pi a}{x^{2}+n^{2}}-\frac{n \sin 2 n \pi a}{x^{2}+n^{2}}\right\}
$$

from which we deduce certain other partial fraction expansions

$$
\frac{1}{e^{\pi x}+1}=\frac{1}{2}+\frac{2}{\pi} \sum_{0}^{\infty} \frac{x}{(2 n+1)^{2}-x^{2}},
$$

$$
\frac{\sinh \pi a x}{\sinh \pi x}=-\frac{2}{\pi} \sum_{1}^{\infty} \frac{(-1)^{n} n}{x^{2}+n^{2}} \sin \pi a n \quad(-1<a<1),
$$

$$
\frac{e^{\pi a x}+e^{\pi(1-a) x}}{e^{\pi x}+1}=\frac{4}{\pi} \sum_{0}^{\infty} \frac{(2 n+1)}{x^{2}+(2 n+1)^{2}} \sin \pi a(2 n+1)
$$$$
(0<a<1)
$$

$2.24 \frac{\cosh (\pi a x / 2)}{\cosh (\pi x / 2)}=\frac{4}{\pi} \sum_{0}^{\infty} \frac{(-1)^{n}(2 n+1)}{x^{2}+(2 n+1)^{2}} \cos (\pi a(2 n+1) / 2)$

$$
(-1<a<1) \text {. }
$$

3. From the last expansion, for example, we see that we can write

$$
\frac{\cosh (\pi a x / 2) \cos (\pi a x / 2)}{\cosh (\pi x / 2) \cos (\pi x / 2)}=\sum_{0}^{\infty} \frac{A_{n}}{(2 n+1)^{4}-x^{4}} \quad(-1<a<1) .
$$


Multiplying across by $2 n+1-x$ and letting $x \rightarrow 2 n+1$, we determine $A_{n}$ and find

3.1

$$
\frac{\cosh (\pi a x / 2) \cos (\pi a x / 2)}{\cosh (\pi x / 2) \cos (\pi x / 2)}
$$

$$
=\frac{8}{\pi} \sum_{0}^{\infty} \frac{(-1)^{n}(2 n+1)^{3}}{(2 n+1)^{4}-x^{4}} \frac{\cosh (\pi a(2 n+1) / 2) \cos (\pi a(2 n+1) / 2)}{\cosh (\pi(2 n+1) / 2)}
$$

Putting $x=0$ yields

$$
1=\frac{8}{\pi} \sum_{0}^{\infty} \frac{(-1)^{n}}{2 n+1} \frac{\cosh (\pi a / 2) \cos (\pi a(2 n+1) / 2)}{\cosh (\pi(2 n+1) / 2)},
$$

therefore, equating coefficients of $a^{4 m}$,

$$
\sum_{0}^{\infty} \frac{(-1)^{n}(2 n+1)^{4 m+3}}{\cosh (\pi(2 n+1) / 2)}=0
$$

The case $m=0$ is series (1.11).

Putting $a=0$ yields

$$
\begin{aligned}
& \frac{1}{\cosh (\pi x / 2) \cos (\pi x / 2)} \\
& =\frac{8}{\pi} \sum_{0}^{\infty} \frac{(-1)^{n}(2 n+1)^{3}}{(2 n+1)^{4}-x^{4}} \frac{1}{\cosh (\pi(2 n+1) / 2)} .
\end{aligned}
$$

Writing

$$
\frac{1}{\cosh x}=1+E_{1} \frac{x}{1 !}+E_{2} \frac{x^{2}}{2 !}+E_{3} \frac{x^{3}}{3 !}+\cdots
$$

then, with the understanding that

$$
E_{n}+\left(\begin{array}{c}
n \\
1
\end{array}\right) E_{n-1} E_{1} \alpha+\left(\begin{array}{l}
n \\
2
\end{array}\right) E_{n-2} E_{2} \alpha^{2}+\cdots=(E+E \alpha)^{n},
$$

we have, on equating coefficients of $x^{4 m}$,

$$
\begin{array}{r}
\sum_{0}^{\infty} \frac{(-1)^{n}}{(2 n+1)^{4 m+1}} \frac{1}{\cosh (\pi(2 n+1) / 2)}=\frac{\pi}{8}\left(\frac{\pi}{2}\right)^{4 m}(E+E i)^{4 m} \\
(m>0) .
\end{array}
$$

The case $m=1$ is series (1.12). 
4. Similarly, we have

4.1

$$
\frac{e^{\pi a x}+e^{\pi(1-a) x}}{e^{\pi x}+1} \frac{e^{\pi a x i}+e^{\pi(1-a) x i}}{e^{\pi x i}+1}=\frac{4}{\pi} \sum_{0}^{\infty} \frac{(2 n+1)^{3}}{(2 n+1)^{4}-x^{4}}
$$

$$
\frac{\left(e^{\pi a(2 n+1)}+e^{\pi(1-a)(2 n+1)}\right)\left(e^{\pi a(2 n+1) i}+e^{\pi(1-a)(2 n+1) i}\right)}{e^{\pi(2 n+1)}+1}(0<a<1) \text {. }
$$

Putting $x=0$ yields

$$
1=\frac{4}{\pi i} \sum_{0}^{\infty} \frac{1}{2 n+1} \frac{\left(e^{\pi a(2 n+1)}+e^{\pi(1-a)(2 n+1)}\right)\left(e^{\pi a(2 n+1) i}+e^{\pi(1-a)(2 n+1) i}\right)}{e^{2 \pi(n+1)}+1} \quad(0<a<1) .
$$

Differentiating with respect to $a$, this becomes

$$
\sum_{0}^{\infty} \frac{\sinh \pi a(2 n+1) \cos \pi a(2 n+1)+\cosh \pi a(2 n+1) \sin \pi a(2 n+1)}{e^{\pi(2 n+1)}+1}
$$

$$
=\frac{1}{4 i}\left(\frac{1-i}{e^{\pi a(1-i)}-e^{-\pi a(1-i)}}-\frac{1+i}{e^{\pi a(1+i)}-e^{-\pi a(1+i)}}\right) \quad(0<a<1) .
$$

Writing

$$
\frac{x}{e^{x}-1}=1+B_{1} \frac{x}{1 !}+B_{2} \frac{x^{2}}{2 !}+B_{3} \frac{x^{3}}{3 !}+\cdots,
$$

then

$$
\begin{aligned}
& \frac{1-i}{e^{y(1-i)}-e^{-y(1-i)}}-\frac{1+i}{e^{y(1+i)}-e^{-y(1+i)}} \\
& =\frac{2 i}{y}\left(2 B_{2}(2-1) \frac{y^{2}}{2 !}-2^{3} B_{6}\left(2^{5}-1\right) \frac{y^{6}}{6 !}+2^{5} B_{10}\left(2^{9}-1\right) \frac{y^{10}}{10 !}-\cdots\right),
\end{aligned}
$$

and since

$$
\sinh y \cos y+\cosh y \sin y=2 \frac{y}{1 !}-2^{3} \frac{y^{5}}{5 !}+2^{5} \frac{y^{9}}{9 !}-\cdots,
$$

therefore

$$
\sum_{0}^{\infty} \frac{(2 n+1)^{4 m+1}}{e^{\pi(2 n+1)}+1}=\frac{1}{2} \frac{B_{4 m+2}}{(4 m+2) !}\left(2^{4 m+1}-1\right) \quad(m \geqq 0) .
$$

The case $m=1$ is formula (1.21).

We also have 
4.31

$$
\left(\frac{1}{e^{\pi x}+1}-\frac{1}{2}\right)\left(\frac{1}{e^{\pi x i}+1}-\frac{1}{2}\right)
$$

$$
=\frac{2 i}{\pi} \sum_{0}^{\infty} \frac{x^{2}(2 n+1)}{(2 n+1)^{4}-x^{4}} \tanh (\pi(2 n+1) / 2) .
$$

Writing

$$
\frac{2}{e^{x}+1}=1+D_{1} \frac{x}{1 !}+D_{2} \frac{x^{2}}{2 !}+D_{3} \frac{x^{3}}{3 !}+\cdots
$$

so that

$$
D_{n}=\left(2-2^{n+2}\right) \frac{B_{n+1}}{n+1}
$$

then

$$
\sum_{0}^{\infty} \frac{\tanh (\pi(2 n+1) / 2)}{(2 n+1)^{4 m+3}}=\frac{\pi^{4 m+3}}{8 i} \frac{(D+D i)^{4 m+2}}{(4 m+2) !} \quad(m \geqq 0) .
$$

The case $m=0$ is series (1.22).

5. Again, from

$5.1 \frac{\sinh \pi a x \sin \pi a x}{\sinh \pi x \sin \pi x}=\frac{4}{\pi} \sum_{1}^{\infty} \frac{(-1)^{n} n^{3}}{x^{4}-n^{4}} \frac{\sinh \pi a n \sin \pi a n}{\sinh \pi n}(-1<a<1)$, we have, on letting $x \rightarrow 0$,

$$
a^{2}=-\frac{4}{\pi} \sum_{1}^{\infty}(-1)^{n} \frac{\sinh \pi a n \sin \pi a n}{n \sinh \pi n} \quad(-1<a<1) .
$$

Since

$$
\sin y \sinh y=2 \frac{y^{2}}{2 !}-2^{3} \frac{y^{6}}{6 !}+2^{5} \frac{y^{10}}{10 !}-\cdots,
$$

therefore

$$
\sum_{1}^{\infty} \frac{(-1)^{n} n^{4 m+1}}{\sinh \pi n}=0
$$

Equating coefficients of $a^{2}$, we have the case $m=0$, i.e. series (1.31). Dividing across by $a$ and letting $a \rightarrow 0$, we have

$$
\frac{x^{2}}{\sinh \pi x \sin \pi x}=\frac{4}{\pi} \sum_{1}^{\infty} \frac{(-1)^{n} n^{5}}{x^{4}-n^{4}} \frac{1}{\sinh \pi n} .
$$


Writing

$$
\frac{x}{\sinh x}=1+C_{1} \frac{x}{1 !}+C_{2} \frac{x^{2}}{2 !}+C_{3} \frac{x^{3}}{3 !}+\cdots,
$$

so that $C_{n}=\left(2-2^{n}\right) B_{n}$, then

$$
\sum_{1}^{\infty} \frac{(-1)^{n}}{n^{4 m-1} \sinh \pi n}=-\frac{\pi^{4 m-1}}{4} \frac{(C+C i)^{4 m}}{(4 m) !} \quad(m>0) .
$$

The case $m=1$ is series (1.32).

6. Lastly, we have

6.1

$$
\frac{e^{2 \pi a x}-e^{2 \pi(1-a) x}}{e^{2 \pi x}-1} \frac{e^{2 \pi a x i}-e^{2 \pi(1-a) x i}}{e^{2 \pi x i}-1}=\frac{2}{\pi i} \sum_{1}^{\infty} \frac{n^{3}}{x^{4}-n^{4}}
$$

$$
\frac{\left(e^{2 \pi a n}-e^{2 \pi(1-a) n}\right)\left(e^{2 \pi a n i}-e^{2 \pi(1-a) n i}\right)}{n\left(e^{2 \pi n}-1\right)} \quad(0<a<1) .
$$

Letting $x \rightarrow 0$, we have

$(2 a-1)^{2}=\frac{2}{\pi i} \sum_{1}^{\infty} \frac{\left(e^{2 \pi a n}-e^{2 \pi(1-a) n}\right)\left(e^{2 \pi a n i}-e^{2 \pi(1-a) n i}\right)}{n\left(e^{2 \pi n}-1\right)}(0<a<1)$.

Differentiating with respect to $a$, this becomes

$$
4(2 a-1)=-16 \sum_{1}^{\infty} \frac{\sin 2 \pi a n \cosh 2 \pi a n+\cos 2 \pi a n \sin 2 \pi a n}{e^{2 \pi n}-1}
$$

6.21

$$
+4\left(\frac{1+i}{e^{2 \pi a(1-i)}-1}+\frac{1-i}{e^{2 \pi a(1+i)}-1}\right)(0<a<1) .
$$

Since

$\sin y \cosh y+\cos y \sinh y=2 \frac{y}{1 !}-2^{3} \frac{y^{5}}{5 !}+2^{5} \frac{y^{9}}{9 !}-\cdots$,

and

$$
\begin{aligned}
& \frac{1+i}{e^{y(1-i)}-1}+\frac{1-i}{e^{y(1+i)}-1} \\
& \quad=-1+\frac{2}{y}\left(2 B_{2} \frac{y^{2}}{2 !}-2^{3} B_{6} \frac{y^{6}}{6 !}+2^{5} B_{10} \frac{y^{10}}{10 !}-\cdots\right),
\end{aligned}
$$

therefore 
6.221

6.222

$$
\begin{array}{ll}
\sum_{1}^{\infty} \frac{n}{e^{2 \pi n}-1}=\frac{1}{24}-\frac{1}{8 \pi}, & \\
\sum_{1}^{\infty} \frac{n^{4 m+1}}{e^{2 \pi n}-1}=\frac{1}{2} \frac{B_{4 m+2}}{4 m+2} \quad(m>0) .
\end{array}
$$

The case $m=3$ is series (1.41).

We also have

$$
\left(\frac{1}{e^{2 \pi x}-1}+\frac{1}{2}\right)\left(\frac{1}{e^{2 \pi x i}-1}+\frac{1}{2}\right)
$$

6.31

$$
=\frac{1}{4 \pi^{2} x^{2} i}+\frac{1}{\pi i} \sum_{1}^{\infty} \frac{n}{x^{4}-n^{4}} \operatorname{coth} \pi n,
$$

therefore

$$
\sum_{1}^{\infty} \frac{\operatorname{coth} \pi n}{n^{4 m-1}}=-\frac{(2 \pi)^{4 m-1}}{2} \frac{(B+B i)^{4 m}}{(4 m) !}, \quad(m>0) .
$$

The case $m=2$ is series (1.42).

\section{REFERENCES}

1. S. Ramanujan, Collected papers of Srinivasa Ramanujan, Cambridge, 1927. The series (1.41), (1.42), (1.12) are theorems (4), (5), (6), page xxxv, and the series (3.22), (6.221) are questions 358,387 , page 326 . Although he only stated some particular cases there is no doubt that all the general formulae of this paper were known to Ramanujan. Those familiar with his work will recall his preference for stating most interesting particular cases rather than writing out general formulae.

Other proofs of some of these series have been given:

2. G. N. Watson, J. London Math. Soc. vol. 3 (1928) pp. 216-225.

3. G. H. Hardy, J. London Math. Soc. vol. 3 (1928) pp. 238-240.

4. H. F. Sandham, Quart. J. Math. (2) vol. 1 (1950) pp. 238-240.

Dublin Institute for Advanced Studies 